\title{
The role of a parental history of Balkan endemic nephropathy in the occurrence of BEN: a prospective study
}

This article was published in the following Dove Press journal: International Journal of Nephrology and Renovascular Disease 3 April 2012

Number of times this article has been viewed

\section{Kesinee Hanjangsit ${ }^{1}$ \\ Wilfried Karmaus' \\ Plamen Dimitrov ${ }^{2}$ \\ Hongmei Zhang' \\ Jim Burch' \\ Svetla Tzolova ${ }^{2}$ \\ Vecihi Batuman ${ }^{3,4}$}

'Department of Epidemiology and Biostatistics, University of South

Carolina, Columbia, SC, USA;

${ }^{2}$ National Center of Public Health

Protection, Sofia, Bulgaria; ${ }^{3}$ Section of Nephrology-Hypertension, Tulane

University Medical Center, New

Orleans, LA, USA; ${ }^{4}$ VA Medical

Center, New Orleans, LA, USA
Correspondence: Wilfried Karmaus Department of Epidemiology and Biostatistics, Arnold School of Public Health, University of South Carolina, 800 Sumter Street, Columbia,

SC 29208, USA

Tel +l 8037779814

Fax + I 8037772524

Email karmaus@sc.edu
Abstract: Balkan endemic nephropathy (BEN) is a chronic kidney disease that affects persons living in the Balkans. Despite the unique geographical specificity of this disease, its etiology has remained unclear. Even if a positive family history of BEN has been identified, it is still uncertain how the disease develops in offspring. In this paper, we examine clinical mechanisms related to the onset of BEN in individuals who have a parental history of BEN to identify early detection of the disease and formulate interventions. We conducted a 5-year prospective study, using markers in years one and three to predict new cases of BEN in year five. New cases of BEN were defined based on three criteria: parental history of BEN, reduced kidney size, and reduced kidney function. Incident cases were divided into (1) probable, (2) definite, and (3) combined labeled total incidence. We evaluated parental history in relation to BEN and tested the potentially intervening effects of kidney length, kidney cortex width, $\beta_{2}$-microglobulin, C-reactive protein, and creatinine clearance, using path analyses. The findings of the path analyses suggested that parental history of BEN had both direct and indirect effects. The direct effect was significant for all three modes of parental history (biparental, maternal, and paternal; odds ratios 71.5, 52.3, and 50.1, respectively). The indirect effects of maternal BEN acted via kidney length and creatinine clearance. Biparental BEN was mediated by (1) kidney length and creatinine clearance, and (2) creatinine clearance alone. Paternal BEN had three indirect effects: (1) through kidney length and creatinine clearance, (2) via kidney cortex width and creatinine clearance, and (3) via kidney cortex width only. In conclusion, a family history of BEN led to reduced kidney length and cortex width, and a decline in creatinine clearance, which in turn predicted the onset of BEN.

Keywords: Balkan endemic nephropathy, incidence of BEN, parental history of BEN, kidney size, $\beta_{2}$-microglobulin, creatinine clearance

\section{Introduction}

Balkan endemic nephropathy $(\mathrm{BEN})$ is a renal disease with an unknown etiology. ${ }^{1,2}$ It solely targets the Balkan population. ${ }^{3-5} \mathrm{BEN}$ is not diagnosed in children but is discovered in victims when they reach their 50 s, suggesting a long latency period. A family history of BEN has been demonstrated to increase the risk of this disease. ${ }^{6,7}$ Additionally, decreased kidney sizes, reduced creatinine clearance rate $(\mathrm{CCr})$, and increased urine excretion, especially of $\beta_{2}$-microglobulin, have been illustrated to be related to BEN. ${ }^{2,8}$

Existing literature and prior studies have demonstrated that increased $\beta_{2}$-microglobulin and reduced kidney length were associated with both BEN patients and their healthy offspring. ${ }^{9-15}$ Moreover, increased C-reactive protein (CRP) and 
narrow kidney cortex width were related to a parental history of BEN (PHB) before the disease manifestation. A reduced $\mathrm{CCr}$ was connected to both BEN patients and offspring, and it was inversely correlated with kidney sizes in BEN patients. ${ }^{9,13,15}$ Even when an affected family has been verified, it is still uncertain how the disease develops in offspring. We hypothesized that kidney length and cortex width, CCr, CRP, or $\beta_{2}$-microglobulin may be intervening factors between $\mathrm{PHB}$ and BEN development. If we can identify morphological and functional changes that lead to BEN in individuals with higher risk due to $\mathrm{PHB}$, we may be able to target specific interventions.

To respond to this challenge, it is necessary to evaluate the effect and direction of biological factors on developmental BEN. One option pursued in this study was firstly to track clinical changes over time in adult offspring of BEN patients, and secondly to identify links in the relationship between PHB and onset of BEN. This would allow us to distinguish whether such changes were in the pathway of the developmental BEN or were only bystanders. We used path analysis to evaluate the various links. This method enabled us to study direct and indirect association among PHB, clinical markers, and incidence of BEN simultaneously as a web of associations. ${ }^{16}$

To our knowledge, no study has investigated the pathways between PHB and the incidence of BEN. Moreover, out of multiple possible markers, we have not identified those that link PHB and onset of BEN. Therefore, a hypothetical path model (Figure 1) was constructed, bridging the links between PHB and new cases of BEN, based upon the literature and prior studies. We tested whether or not PHB, which leads to new onset of BEN in offspring, is mediated by kidney size, function, or serum levels of CRP. If effects of PHB were mediated, then intervening variables would be identified. If none of the tested morphological and functional changes statistically significantly link PHB with the onset of BEN, we would conclude that no potential mediators could be detected.

\section{Materials and methods Study design and population}

A follow-up study in healthy adult offspring from families with and without BEN was conducted in Bulgaria and is described in detail elsewhere. ${ }^{10,17}$ Briefly, 104 adult offspring with a PHB were recruited from a hospital database in Vratza, where BEN is still endemic. Ninety-seven adult offspring of non-BEN parents were frequency-matched with regard to sex and 10-year age groups and served as the control group. Both study groups were enrolled and examined for the first time in 2003-2004 and reexamined four times between 2004 and 2008. In 2005-2006, 18 participants were newly recruited with offspring of BEN patients. The institutional review boards of the National Center of Public Health Protection, Sofia, Bulgaria, and of the University of South Carolina approved the study. Participants provided written consent.

\section{Data collection}

Face-to-face interviews were conducted asking participants about their birth date and family history of BEN. Both kidneys' sizes were measured using ultrasound. The longest dimension and the smallest thickness of kidney parenchyma were determined. Average length and cortex width of both kidneys were calculated. $\beta_{2}$-microglobulin was measured in an aliquot of the hour-one urine sample, and details have

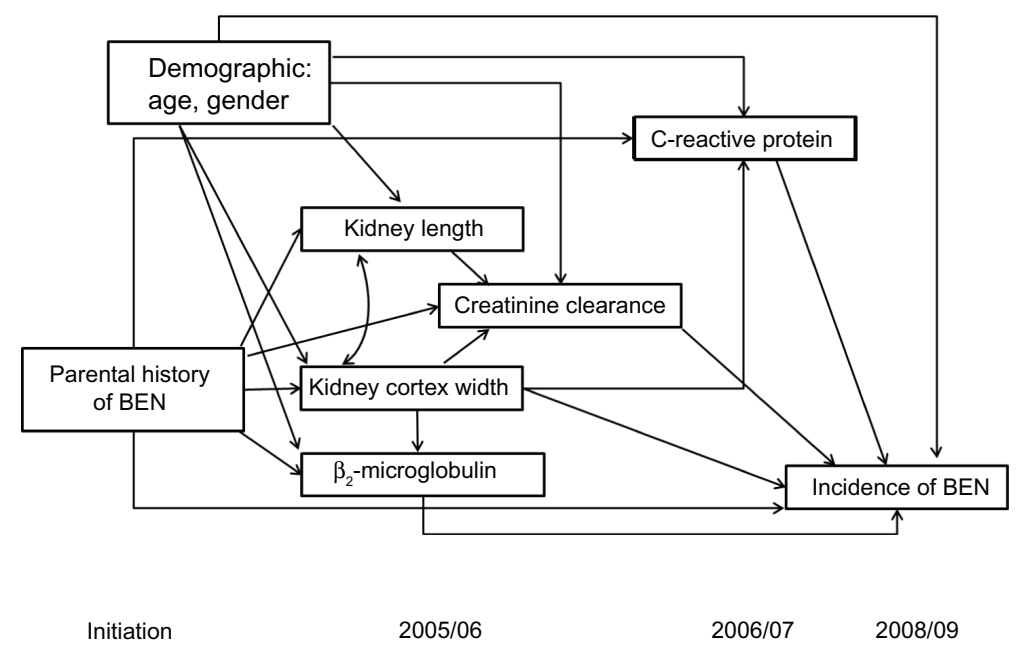

Figure I Hypothesized path diagram.

Note: Arrows represent the effect of one variable on another.

Abbreviation: BEN, Balkan endemic nephropathy. 
been described elsewhere. ${ }^{10} \mathrm{CCr}$ was estimated from serum creatinine values by using the Cockcroft-Gault formula ${ }^{18}$ and then normalized to body surface area. Serum CRP was measured with Immulite (Siemens, Tarrytown, NY) chemiluminescent immunometric assay. ${ }^{17}$

\section{Definition of incidence of BEN}

Definite cases of BEN required (1) PHB, (2) reduced kidney size (kidney length $<100 \mathrm{~mm}$, kidney cortex width $<11 \mathrm{~mm}$ ), and (3) reduced kidney functions (serum creatinine $>124 \mu \mathrm{mol} / \mathrm{L}$ for males and $>106 \mu \mathrm{mol} / \mathrm{L}$ for females; creatinine clearance using the Cockcroft-Gault formula $<60 \mathrm{~mL} / \mathrm{min}$; and $\beta_{2}$-microglobulin $>0.2 \mathrm{mg} / \mathrm{g}$ creatinine). Probable BEN cases were defined as those with PHB, reduction in either kidney length or cortex width, and reduction in at least one kidney function marker with repeated measurements. Both groups were combined as the incidence of BEN. The criteria we applied were central criteria used in previous studies. ${ }^{2,6,8}$

\section{Statistical analysis}

Descriptive statistics were applied to characterize the study population. To test the prediction of BEN in 2008-2009, age and clinical markers, comprising kidney length and cortex width, CCr, $\beta_{2}$-microglobulin in 2003-2004 and 2005-2006, and CRP in 2006-2007, were used as continuous variables. Due to a skewed distribution, $\beta_{2}$-microglobulin and CRP were log-transformed. In the path analysis, the independent variable 'PHB' was analyzed in two ways: (1) as the specific status of PHB (biparental, maternal, parental, and non-BEN); and (2) as the dichotomous outcome (yes/no). An ordinal BEN classification (definite, probable, and non-BEN) was used as outcome variable. To determine the agreement between repeated measurements, we estimated the intraclass correlation coefficient (ICC). ${ }^{19}$

To test whether kidney sizes, functions, or CRP mediate the path from PHB while controlling for age and sex, path analyses were applied using Mplus 6 (Muthén and Muthén, Los Angeles, CA). ${ }^{20}$ This method distinguishes three types of effects: direct, indirect, and total. Direct effects indicate the impact of a risk factor on an outcome not mediated by other variables. In Mplus 6, the indirect effect is calculated by the product of the coefficient method. ${ }^{21}$ The total effect of a risk factor is the sum of direct and indirect statistical links. ${ }^{16}$

Our results showed that PHB perfectly predicted the occurrence of BEN and caused the maximum likelihood estimate for the effect of PHB infinitely. Penalized maximum likelihood estimation could be used to resolve this problem. ${ }^{22}$ However, since it is not provided in Mplus 6, to ease the computing burden, one pseudo-BEN case was generated by a random selection among the non-BEN subjects with no parental history.

Estimates in Mplus 6 for incidence of BEN are shown as logit coefficients in the path analyses and are transformed to odds ratios $(\mathrm{ORs}=\exp$ [logit coefficient]) to explain the strength of the association. Regarding kidney length and cortex width, $\mathrm{CCr}, \mathrm{CRP}$, and $\beta_{2}$-microglobulin, the estimation of coefficients are linear regression coefficients. ${ }^{20}$ Per unit increase in the independent variable, the effect is estimated by multiplying the estimated path coefficient with the unit increase in the dependent variable.

Mplus 6 provides the Akaike information criterion and Bayesian information criterion as indicators of the model fit; the smaller the model indicators, the better the fit. ${ }^{20}$ Selecting a model involves simultaneously fitting a series of linear regressions and logistic regressions and then choosing the model for the best fit.

\section{Results}

A total of 219 participants were enrolled in this study, and 177 $(80.8 \%)$ remained in the last year of follow-up. Over a 5-year period, 38 (17.4\%) offspring with a parental history of BEN developed the disease, of which $6.8 \%$ and $10.5 \%$ developed definite and probable BEN, respectively, whereas offspring without a parental history of BEN did not develop BEN.

Participants with definite BEN were older, had the smallest kidney length and cortex width, and presented high $\beta_{2}$-microglobulin levels (Table 1). Moreover, about $50 \%$ of participants with definite BEN had a maternal history of BEN. Definite BEN was equally frequent in women and men. In the paternal BEN group, more probable BEN cases were found. Women were slightly more likely to develop probable BEN (Table 1).

Regarding rank correlations of age and clinical markers, most were moderately correlated with age and each other $(P<0.05$, Table 2). Maternal BEN was correlated with kidney length $(P=0.009)$. A high agreement between measurements of kidney length and cortex width in left and right kidneys (ICC $=0.99$ ) over the course of two repeated assessments was detected ( $\mathrm{ICC}=0.98$, data not shown). Regarding kidney function ( $\mathrm{CCr}$ and $\beta_{2}$-microglobulin), the ICCs over the course of two repeated examinations were 0.99 for both markers.

\section{Model with PHB (yes/no) as predictor}

Statistically significant path coefficients for this model are presented in Figure 2. Single-headed arrows in the path 
Table I Characteristics of participants stratified by incidence of BEN in year 2005-2006

\begin{tabular}{|c|c|c|c|}
\hline Variables & $\begin{array}{l}\text { Definite BEN } \\
(n=15) \\
N \text { cases }(\%)\end{array}$ & $\begin{array}{l}\text { Probable BEN } \\
(n=23) \\
\mathbf{N} \text { cases }(\%)\end{array}$ & $\begin{array}{l}\text { No BEN } \\
(n=162) \\
N \text { cases }(\%)\end{array}$ \\
\hline \multicolumn{4}{|l|}{ Sex } \\
\hline Female & $8(53.33)$ & $14(60.87)$ & $84(51.85)$ \\
\hline \multicolumn{4}{|l|}{ Parental history of BEN } \\
\hline Biparental & $5(33.33)$ & $8(34.78)$ & $21(12.96)$ \\
\hline Mother & $8(53.33)$ & $6(26.09)$ & $26(16.05)$ \\
\hline Father & $2(13.33)$ & $9(39.13)$ & $23(14.20)$ \\
\hline \multicolumn{4}{|l|}{ Living in endemic area } \\
\hline$<20$ years & $0(0.00)$ & $2(8.70)$ & $22(13.58)$ \\
\hline $21-40$ years & $3(20.00)$ & $3(13.04)$ & $64(39.51)$ \\
\hline$\geq 41$ years & $12(80.00)$ & $18(78.86)$ & $76(46.91)$ \\
\hline \multicolumn{4}{|l|}{ Body mass index } \\
\hline Overweight & II (73.33) & $10(43.48)$ & 69 (42.59) \\
\hline Obesity & $2(13.33)$ & $9(39.13)$ & $42(25.93)$ \\
\hline \multicolumn{4}{|l|}{ History of other kidney disease } \\
\hline Pyelonephritis & $3(20.00)$ & $2(8.70)$ & $8(4.97)$ \\
\hline Other (stone, cystitis, cancer) & $3(20.00)$ & $4(17.39)$ & $22(13.66)$ \\
\hline \multicolumn{4}{|l|}{ Smoking status } \\
\hline Current & $4(26.67)$ & $4(17.39)$ & $60(37.64)$ \\
\hline Exsmoker & I (6.67) & $0(0.00)$ & $4(2.47)$ \\
\hline \multicolumn{4}{|l|}{ Mean ( $95 \% \mathrm{Cl}$, or $5 \%, 95 \%)$} \\
\hline Age (year) & $61.26(56.79,65.74)$ & $54.78(51.46,58.1 \mathrm{I})$ & $49.69(48.26,51.10)$ \\
\hline Kidney length $(\mathrm{mm})$ & $109.88(102.64,117.02)$ & II $3.20(109.74,116.64)$ & $119.03(117.94,120.13)$ \\
\hline Kidney cortex width (mm) & $12.99(11.72,14.25)$ & $14.65(13.81,16.65)$ & $16.07(15.79,16.35)$ \\
\hline $\mathrm{CCr}\left(\mathrm{mL} / \mathrm{min} / \mathrm{I} .73 \mathrm{~m}^{2}\right)$ & $73.23(62.68,81.78)$ & $83.67(78.80,88.65)$ & $91.03(88.42,93.65)$ \\
\hline $\mathrm{CRP}^{\mathrm{a}}(\mathrm{mg} / \mathrm{dL})$ & $0.50(0.08,3.43)$ & $0.44(0.09,1.7 \mathrm{I})$ & $0.27(0.06,1.73)$ \\
\hline$\beta_{2} M^{a}(\mu g / g$ creatinine $)$ & I $23.56(75.77,892.29)$ & II $4.89(38.72,800.00)$ & $108.02(47.84,363.28)$ \\
\hline
\end{tabular}

Note: Median.

Abbreviations: $\mathrm{CCr}$, creatinine clearance rate; $\mathrm{BEN}$, Balkan endemic nephropathy; $\beta_{2} \mathrm{M}, \beta_{2}$-microglobulin; CRP, C-reactive protein.

diagrams demonstrate the effect of a PHB on outcome variables (kidney length, kidney cortex width, $\mathrm{CCr}$, or incidence of BEN). Single-headed arrows with a solid line represent a direct effect while those with a dotted line demonstrate an indirect effect. The curved line with arrows at each end indicates a correlation between variables. The value on each line represents the path coefficient.

Participants with a PHB were associated with small kidney length and cortex width and increased CRP level. A reduction of the kidney cortex width of $10 \mathrm{~mm}$ increased $\beta_{2}$-microglobulin by about $6 \mu \mathrm{g} / \mathrm{g}$ creatinine. There were positive associations between $\mathrm{CCr}$ and both kidney length and cortex width in all participants. No association was identified between PHB and $\beta_{2}$-microglobulin or between CRP and either kidney size or function. Kidney cortex width, $\mathrm{CCr}$, and PHB were directly related to the occurrence of BEN. Offspring with a PHB were 57 times more likely to develop BEN than persons without a PHB (exponential of 4.05 , Figure 2). A reduction of kidney cortex width of $1 \mathrm{~mm}$ increased the risk of having BEN $(\mathrm{OR}=0.74$; exponential of -0.29). Furthermore, a decline in $\mathrm{CCr}$ was associated with the occurrence of $\mathrm{BEN}(\mathrm{OR}=0.94$; exponential of -0.06$)$. No direct associations between CRP, kidney length, or $\beta_{2}$-microglobulin and new cases of BEN were detected. We also found that PHB was indirectly associated with the occurrence of BEN through decreased kidney length or kidney cortex width and reduced CCr. Another pathway was mediated by kidney cortex width alone. When all variables were included, $64.3 \%$ of the incidence of BEN was explained.

\section{Model with specific status of PHB as predictor}

When examining the model using the specific PHB, we found that short kidney length and increased CRP level were related to participants with a maternal history of BEN (Figure 3). Increased $\mathrm{CCr}$ and CRP levels in participants were related to a biparental history of BEN. No association was identified between any PHB and $\beta_{2}$-microglobulin or between CRP and either kidney size or function.

Statistically significant direct associations were observed between the incidence of BEN and all three PHBs, indicating that a major part of the effect of PHB on the onset of BEN 
Table 2 Rank correlation coefficients (Spearman) of parental history, age, and clinical markers

\begin{tabular}{|c|c|c|c|c|c|c|c|c|c|}
\hline & \multicolumn{5}{|l|}{$n=219$} & \multirow{2}{*}{$\frac{n=198}{C R P}$} & \multicolumn{3}{|l|}{$n=219$} \\
\hline & $\begin{array}{l}\text { Age } \\
\text { (years) }\end{array}$ & $\begin{array}{l}\text { Kidney cortex } \\
\text { width }\end{array}$ & $\begin{array}{l}\text { Kidney } \\
\text { length }\end{array}$ & $\mathrm{CCr}$ & $\beta_{2} M$ & & Biparental & Mother & Father \\
\hline Age (year) & 1.00 & & & & & & & & \\
\hline Kidney cortex width & $-0.421^{*}$ & 1.00 & & & & & & & \\
\hline Kidney length & $-0.192 *$ & $0.363^{*}$ & 1.00 & & & & & & \\
\hline $\mathrm{CCr}$ & $-0.64 I^{*}$ & $0.458^{*}$ & $0.294^{*}$ & 1.00 & & & & & \\
\hline$\beta_{2} M$ & $0.28 I^{*}$ & $-0.273^{*}$ & 0.003 & $-0.212^{*}$ & 1.00 & & & & \\
\hline CRP & $0.236 *$ & $-0.146^{*}$ & -0.015 & -0.113 & $0.07 \mid$ & 1.00 & & & \\
\hline Biparental & $0.183^{*}$ & -0.067 & -0.084 & -0.040 & -0.045 & $0.179 *$ & 1.00 & & \\
\hline Mother & $0.133^{*}$ & -0.047 & $-0.176^{*}$ & -0.093 & 0.072 & 0.131 & $-0.237^{*}$ & 1.00 & \\
\hline Father & $-0.181^{*}$ & -0.066 & -0.012 & 0.051 & -0.029 & -0.038 & $-0.127^{*}$ & $-0.237 *$ & 1.00 \\
\hline
\end{tabular}

Note: $* P$ value $<0.05$.

Abbreviations: $\mathrm{CCr}$, creatinine clearance rate; $\mathrm{BEN}$, Balkan endemic nephropathy; $\beta_{2} \mathrm{M}, \beta_{2}$-microglobulin; CRP, C-reactive protein.

was not mediated by clinical markers. Those offspring with a biparental BEN status were 71.5 times more likely to develop BEN than those without a PHB (OR = exponential of 4.27, Figure 3). Participants with a maternal history of BEN were 52.3 times more likely to develop BEN, while those with a paternal history of BEN were 50.1 times more likely to develop the disease than those without a PHB (OR = exponential of 3.91). A reduction of kidney cortex width of $1 \mathrm{~mm}$ increased the risk of having $\mathrm{BEN}(\mathrm{OR}=0.75$; exponential of -0.28$)$. Also, an increase in $\mathrm{CCr}$ was associated with lower occurrence of $\mathrm{BEN}(\mathrm{OR}=0.94$; exponential of -0.059$)$. No direct associations between CRP, kidney length, or $\beta_{2}$-microglobulin and new cases of BEN were detected.

There were several indirect impacts of PHB on the onset of BEN (Figure 3). We found that maternal history of BEN had an indirect effect on BEN through reduced kidney length, which then affected CCr. Paternal history of BEN had three indirect effects on developing BEN: mediated by kidney length and $\mathrm{CCr}$, by kidney cortex width and $\mathrm{CCr}$, and by kidney cortex width alone. The indirect paths of biparental history on BEN were mediated by two clinical markers (kidney length and $\mathrm{CCr}$ ) and $\mathrm{CCr}$ only. Overall, the model accounted for $67.3 \%$ of the variability in the incidence of BEN.

For both models in Figures 2 and 3, age and sex were not associated with the occurrence of BEN; however, an increase in age and being female were statistically significantly associated with almost all clinical markers. Thus, both factors indirectly affected BEN via $\mathrm{CCr}$ or kidney cortex width. All models fit well, with the smallest of the Akaike information

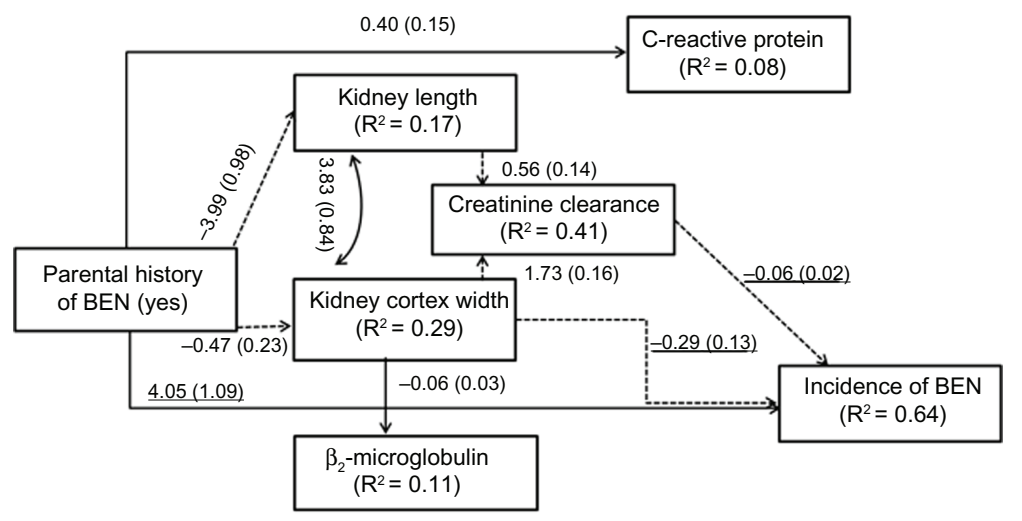

Initiation 2005/06 2008/09

Figure 2 Direct and indirect path linking parental Balkan endemic nephropathy (BEN) and the incidence of BEN.

Notes: The unstandardized (standard error) parameter estimates are shown. The dotted line presents a significant statistic $(P<0.05)$ of an indirect association between parental history of BEN and the occurrence of BEN. The solid line shows a statistically significant direct effect between parental history of BEN and the occurrence of BEN $(P<0.05)$. The underlined coefficients are logit coefficients and need to be exponentiated to calculate odds ratios. The nonunderlined coefficients are interpreted as linear regression coefficients: per unit of increase in the independent variable there will be a coefficient unit of increase in the dependent variable. The curved line with arrows at each end indicates a correlation between variables. 


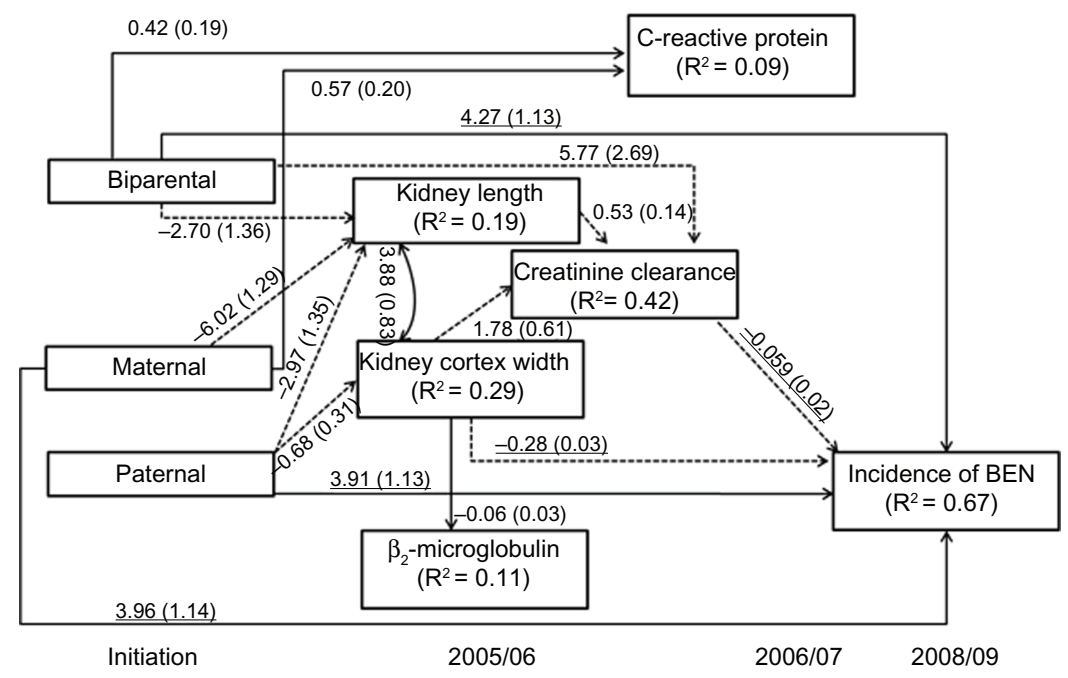

Figure 3 Direct and indirect path linking a specific status of parental Balkan endemic nephropathy (BEN) and the incidence of BEN.

Notes: The unstandardized (standard error) parameter estimates are shown. The dotted line presents a significant statistic $(P<0.05)$ of an indirect association between parental history of BEN and the occurrence of BEN. The solid line shows a statistically significant direct effect between parental history of BEN and the occurrence of BEN $(P<0.05)$. The underlined coefficients are logit coefficients and need to be exponentiated to estimate odds ratios. The nonunderlined coefficients are interpreted as linear regression coefficients: per unit of increase in the independent variable there will be a coefficient unit of increase in the dependent variable. The curved line with arrows at each end indicates a correlation between variables.

criterion and Bayesian information criterion. The goodness of fit was high for all models.

\section{Discussion}

This paper aimed to examine whether the association between PHB and incidence of BEN was mediated by such clinical markers as kidney sizes, kidney functions, and serum CRP. The results showed that PHB to a large extent directly influenced new occurrence of BEN. However, the effect of PHB on new cases was partially mediated through kidney sizes, kidney cortex width, and CCr. Maternal history influenced the occurrence of new cases via kidney length and $\mathrm{CCr}$. Biparental BEN had two pathways: through kidney length and $\mathrm{CCr}$, and through $\mathrm{CCr}$ only. There were three indirect associations between paternal history of BEN and new onset: (1) small kidney length resulting in decreased $\mathrm{CCr}$, (2) small kidney cortex width and decline in $\mathrm{CCr}$, and (3) declined kidney cortex width. The web of associations also indicated that $\mathrm{CCr}$ was an important intervening factor between parental history of BEN and new cases; however, CRP and $\beta_{2}$-microglobulin did not act as intervening variables.

Our findings are unlikely to have resulted from a selection bias, as a high follow-up proportion of participants remained at the end of study (81\%). Information bias, if any, was minimal, since identical diagnosis criteria of BEN were applied to detect the outcome during the follow-up period, and there was a high agreement of measurements of clinical markers over time, such as kidney dimensions, function, and $\mathrm{CCr}$.
We found that short kidney length was indirectly associated with BEN through decreased CCr. Kidney cortex width directly influenced the onset of BEN and was also mediated through $\mathrm{CCr}$. Our results demonstrated that kidney sizes had a direct effect (kidney cortex width) and an indirect effect (kidney length). These findings support autopsy studies of BEN patients indicating cortical sclerosing atrophy in the early stage of BEN. ${ }^{23,24}$

In agreement with the literature, a decrease of $\mathrm{CCr}$ directly increased the risk of BEN. ${ }^{9,25}$ Other studies have also reported that $\beta_{2}$-microglobulin excretion was higher in BEN patients and their offspring, ${ }^{10,12}$ yet we did not detect an association between $\beta_{2}$-microglobulin, parental history, and the development of BEN. One explanation for this difference is that $\beta_{2}$-microglobulin may not be a part of the pathological development but only a bystander or may be increased after the disease manifests, resulting from a reduced kidney cortex width.

Unexpectedly, CRP was neither directly nor indirectly related to BEN. However, we found that CRP was associated with parental history, agreeing with a prior study. ${ }^{17}$ Since a PHB is an important risk factor for BEN, it is possible that other inflammatory pathways are involved in the development of BEN. Further studies on inflammatory markers are warranted.

The present study focuses on PHB instead of family history, since PHB is less likely to create information biases compared to the collection of the history of BEN in 
grandparents or other relatives. As expected, PHB had a direct effect on the onset of BEN, indicating that PHB is an important risk factor. ${ }^{26,27}$ Participants with biparental history were more likely to develop BEN than those with a maternal or paternal history. ${ }^{1}$

The association between PHB and incidence of BEN seems to have a variety of different pathways (Figure 3). Maternal BEN acted through kidney length, paternal BEN acted via kidney length and kidney cortex width, and biparental $\mathrm{BEN}$ indirectly affected the incidence of BEN via kidney length and $\mathrm{CCr}$. It is likely that kidney sizes and $\mathrm{CCr}$ are significant mediators between parental status and development of BEN. Further studies specifying the web of associations between parental BEN, risk factors, and the incidence of BEN are needed.

It has been documented in healthy persons that kidney sizes are associated with renal parenchymal mass and the total number of nephrons and that their number decreases with age, ${ }^{28,29}$ resulting in the reduction of kidney cortex width and decrease in kidney function. ${ }^{30}$ In the case of BEN, it is possible that offspring with BEN may have a small kidney size at birth due to a reduction in the number of nephrons, ${ }^{31}$ suggesting a maternal influence as demonstrated before. ${ }^{10}$ This assumption is based on the idea that the onset of BEN occurs in childhood, has a long latency period, and then clinically manifests itself at a later age. ${ }^{4}$ There is a need to determine kidney size of BEN offspring at birth or early in life and then follow them up frequently.

Since $64.3 \%$ of the incidence of BEN was explained, our findings support the hypothesized model. However, this does not exclude the possibility of other causal models and additional risk factors. For example, other clinical markers and possible exposures to environmental factors could be included in future path models to improve the explanation of BEN. Given that a reduction of kidney length, kidney cortex width, and $\mathrm{CCr}$ played a central role in mediating parental status of BEN with new occurrence of BEN in offspring, it is important to intervene by preventing a reduction of these markers due to other risk factors such as smoking and obesity. ${ }^{32-34}$

\section{Conclusion}

Our findings provide evidence elucidating the relationship between parental history of BEN (PHB) and incidence of BEN. First, we demonstrated that parental history has both a direct and an indirect effect. Second, we found several path links between PHB and occurrence of BEN: (1) maternal BEN acting via kidney length, (2) paternal BEN acting via kidney length or cortex width, and (3) biparental BEN acting via kidney length or $\mathrm{CCr}$. Third, we demonstrated that $\beta_{2}$-microglobulin excretion and CRP were bystanders and not involved in the pathway. Our results suggest the need for additional studies to corroborate our findings and to explore further the effect of additional factors on the development of BEN.

\section{Acknowledgments}

This research was supported by NIH Research Grant R01 TW06192, funded by the Fogarty International Center and the National Institute of Environmental Health Sciences, National Institutes of Health, USA, awarded to the National Center of Public Health Protection, Sofia, Bulgaria. The authors thank Susan Davis for suggestions on this manuscript.

\section{Disclosure}

The authors report no conflicts of interest in this work.

\section{References}

1. Toncheva D, Dimitrov T, Stojanova S. Etiology of Balkan endemic nephropathy: a multifactorial disease? Eur J Epidemiol. 1998; 14(4):389-394.

2. Stefanovic V, Jelakovic B, Cukuranovic R, et al. Diagnostic criteria for Balkan endemic nephropathy: proposal by an international panel. Ren Fail. 2007;29(7):867-880.

3. Tanchev Y, Dorossiev D. The first clinical description of Balkan endemic nephropathy (1956) and its validity 35 years later. IARC Sci Publ. 1991;115:21-28.

4. Dimitrov T. Balkan endemic nephropathy in Bulgaria. Facta Univ Ser Med Biol. 2002;9(1):7-14.

5. Maríc I, Bukvíc D, Bogdanovíc M, et al. Cross-sectional study in the Balkan endemic nephropathy village of Vreoci (Serbia). BANTAO J. 2006;4(2):5-8.

6. Stefanovic V, Cosyns J. Balkan Nephropathy. Oxford: Oxford University Press; 2004.

7. Stefanovi V, Polenakovi M. Fifty years of research in Balkan endemic nephropathy: where are we now? Nephron Clin Pract. 2009;112(2): c51-c56.

8. Schiller A, Gusbeth-Tatomir P, Pavlovic N, Ferluga D, Spasovski G, Covic A. Balkan endemic nephropathy: a still unsolved puzzle. J Nephrol. 2008;21(5):673-680.

9. Bukvi D, Mari I, Arsenovi A, Jankovi S, Djukanovi L. Prevalence of Balkan endemic nephropathy has not changed since 1971 in the Kolubara region in Serbia. Kidney Blood Press Res. 2007;30:117-123.

10. Dimitrov P, Tsolova S, Georgieva R, et al. Clinical markers in adult offspring of families with and without Balkan Endemic Nephropathy. Kidney Int. 2006;69(4):723-729.

11. Hanjangsit K, Dimitrov P, Karmaus W, et al. Reduced kidney size in adult offspring of Balkan endemic nephropathy patients and controls: a prospective study. Am J Med Sci. 2010;340(2):94-102.

12. Stefanovic V, Mitic-Zlatkovic M, Cukuranovic R, Miljkovic P, Pavlovic N, Vlahovic P. $\beta_{2}$-microglobulin in patients with Balkan nephropathy and in healthy members of their families. Kidney Int Suppl. 1991;40(34):S21-S26.

13. Djukanovic L, Bukvic D, Maric I. Creatinine clearance and kidney size in Balkan endemic nephropathy patients. Clin Nephrol. 2004; 61:384-386. 
14. Radonic M, Radosevic Z. Clinical features of Balkan endemic nephropathy. Food Chem Toxicol. 1992;30(3):189-192.

15. Arsenovic A, Bukvic D, Trbojevic S, Maric I, Djukanovic L. Detection of renal dysfunctions in family members of patients with Balkan endemic nephropathy. Am J Nephrol. 2005;25(1):50-54.

16. Bollen AK. Stucture Equations with Latent Variables. New York, NY: Wiley-Interscience Publication; 1989.

17. Karmaus W, Dimitrov P, Simeonov V, Tsolova S, Batuman V. Offspring of parents with Balkan Endemic Nephropathy have higher C-reactive protein levels suggestive of inflammatory processes: a longitudinal study. BMC Nephrol. 2009;10:10.

18. Cockcroft DW, Gault MH. Prediction of creatinine clearance from serum creatinine. Nephron. 1976;16(1):31-41.

19. Armstrong B, White E, Saracci R. Principles of exposure measurement in epidemiology. In: Armstrong B, White E, Saracci R, editors. Monographs in Epidemiology and Biostatistics. Vol 21. New York, NY: Oxford University Press; 1992.

20. Muthén L, Muthén B, editors. Mplus User's Guide. 6th ed. Los Angeles, CA: Muthén and Muthén; 2010.

21. MacKinnon D, Lockwood C, Brown C, Wang W, Hoffman J. The intermediate endpoint effect in logistic and probit regression. Clin Trials. 2007;4(5):499.

22. Heinze G, Schemper M. A solution to the problem of separation in logistic regression. Stat Med. 2002;21(16):2409-2419.

23. Ferluga D, Hvala A, Trnacevic S, et al. Pathology of Balkan endemic nepropathy: a correlation with established kidney disease entities. Facta Univ Ser Med Biol. 2002;9(1):82-87.

24. Savin M, Bumbaširevi V, Djukanovi L, Petroni V. The significance of apoptosis for early diagnosis of Balkan nephropathy. Nephrol Dial Transplant. 2001;16 Suppl 6:30.
25. Mitic-Zlatkovic M, Cukuranovic R, Lecic N, Stefanovic V. Urinary creatinine excretion in children from families with Balkan endemic nephropathy: evidence for genetic predisposition to the disease. Pathol Biol (Paris). 2000;48(6):554-557.

26. Toncheva D, Dimitrov T. Genetic predisposition to Balkan endemic nephropathy. Nephron. 1996;72(4):564-569.

27. Marinkovic D, Cvjeticanin S. Population-genetic study of Balkan endemic nephropathy in Serbia. Russ J Genet. 2007;43(8):942-946.

28. Maertens S, Van Den Noortgate N. Kidney in old age. Acta Clin Belg. 2008;63(1):8.

29. Schlanger L. Chapter 4: Kidney senescence. In: American Society of Nephrology. Geriatric Nephrology Curriculum. 2009:1-7.

30. Hekmatnia A, Yaraghi M. Sonographic measurement of absolute and relative renal length in healthy Isfahani adults. $J$ Res Med Sci. 2004;9(2):54

31. Lackland D. Mechanisms and fetal origins of kidney disease. JAm Soc Nephrol. 2005;16(9):2531.

32. Culleton BF, Larson MG, Wilson PWF, et al. Cardiovascular disease and mortality in a community-based cohort with mild renal insufficiency. Kidney Int. 1999;56(6):2214-2219.

33. Haroun MK, Jaar BG, Hoffman SC, et al. Risk factors for chronic kidney disease: a prospective study of 23,534 men and women in Washington County, Maryland. J Am Soc Nephrol. 2003;14(11):2934.

34. Levey AS, Atkins R, Coresh J, et al. Chronic kidney disease as a global public health problem: approaches and initiatives - a position statement from Kidney Disease Improving Global Outcomes. Kidney Int. 2007;72(3):247-259.

\section{Publish your work in this journal}

The International Journal of Nephrology and Renovascular Disease is an international, peer-reviewed open-access journal focusing on the pathophysiology of the kidney and vascular supply. Epidemiology, screening, diagnosis, and treatment interventions are covered as well as basic science, biochemical and immunological studies. The journal welcomes original research, clinical studies, reviews \& evaluations, expert opinion and commentary, case reports and extended reports. The manuscript management system is completely online and includes a very quick and fair peerreview system, which is all easy to use. Visit http://www.dovepress.com/ testimonials.php to read real quotes from published authors. 